Differential Equations / Optimal control

\title{
Controllability for a class of reaction-diffusion systems: generalized Kalman's condition
}

\author{
Ammar-Khodja Farid ${ }^{\mathrm{a}}$, Benabdallah Assia ${ }^{\mathrm{b}}$, Dupaix Cédric ${ }^{\mathrm{a}}$, \\ González-Burgos Manuel ${ }^{\mathrm{c}}$,
}

${ }^{a}$ Lab. de Mathématiques UMR 6623, Université de Franche-Comté, 16 rte de Gray, 25030 Besancon cedex, France

${ }^{\mathrm{b}}$ CMI-LATP, UMR 6632, Université de Provence, Technopôle Château-Gombert, 39 rue F. Joliot Curie, 13453 Marseille Cedex 13, France

${ }^{\mathrm{c}}$ Dpto. de Ecuaciones Diferentiales y Análisis Numérico, Universidad Sevilla, Aptdo. 1160, 41080 Sevilla, Spain

Received $* * * * *$; accepted after revision +++++

Presented by

\begin{abstract}
In this article, we study the controllability of a class of parabolic systems of the form $Y_{t}=(D \Delta+A) Y+B \chi_{\omega} u$ with Dirichlet conditions on the boundary of a bounded domain $\Omega$, where $\omega \subset \Omega$ is a subdomain. Here $D, A \in \mathcal{L}\left(\mathbb{R}^{n}\right)$, $B \in \mathcal{L}\left(\mathbb{R}^{m} ; \mathbb{R}^{n}\right)$ and we prove that the algebraic Kalman condition extends to such systems. To cite this article: A. Name1, A. Name2, C. R. Acad. Sci. Paris, Ser.
\end{abstract}

\section{Résumé}

Dans cet article, on étudie la contrôlabilité d'une classe de systèmes paraboliques de la forme $Y_{t}=(D \Delta+A) Y+$ $B \chi_{\omega} u$ avec des conditions de Dirichlet sur le bord d'un domaine born $\Omega$, où $\omega \subset \Omega$ est un sous-domaine. Ici $D, A \in \mathcal{L}\left(\mathbb{R}^{n}\right), B \in \mathcal{L}\left(\mathbb{R}^{m} ; \mathbb{R}^{n}\right)$ et on montre que la condition algbrique de Kalman s'étend à de tels systèmes. Pour citer cet article: A. Name1, A. Name2, C. R. Acad. Sci.Paris, .

\section{Version française abrégée}

Le critère de Kalman est une condition nécessaire et suffisante de contrôlabilité de systèmes différentiels linéaires à coefficients constants. Ce travail a pour objectif de donner une extension de ce critère aux systèmes d'équations aux dérivées partielles, plus précisément aux systèmes paraboliques linéaires de la forme (1) dont les coefficients sont indépendants du temps.

Email addresses: fammarkh@univ-fcomte.fr (Ammar-Khodja Farid), assia@cmi.univ-mrs.fr (Benabdallah Assia), cedric.dupaix@univ-fcomte.fr (Dupaix Cédric), manoloburgos@us.es (González-Burgos Manuel). 
La contrôlabilité de systèmes paraboliques est étudiée depuis les années 2000, essentiellement pour des systèmes de deux équations (voir [11], [1], [2], [5]). Le résultat principal de ce travail est de démontrer que la condition de Kalman (dans un sens à préciser) est nécessaire et suffisante pour la contrôlabilité aux trajectoires de systèmes (1) satisfaisant aux hypothèses suivantes : $\Omega \subset \mathbb{R}^{d}$ est un ouvert borné régulier, $\omega \subset \Omega$ est sous-ensemble ouvert de $\Omega, T>0, M=P D P^{-1}$, où $D=\operatorname{diag}\left(d_{i i}>0\right)_{n \times n} \in \mathcal{L}\left(\mathbb{R}^{n}\right)$, $R$ un opérateur elliptique autoadjoint donné par (2), $A=\left(a_{i j}\right)_{1 \leq i, j \leq n} \in \mathcal{L}\left(\mathbb{R}^{n}\right), B \in \mathcal{L}\left(\mathbb{R}^{m}, \mathbb{R}^{n}\right), v \in$ $L^{2}\left(\Omega_{T}\right)^{m}\left(\Omega_{T}=(0, T) \times \Omega\right), y_{0}=\left(y_{0, i}\right)_{1 \leq i \leq n} \in L^{2}(\Omega)^{n}$.

Dans (1), $y=\left(y_{i}\right)_{1 \leq i \leq n}$ est la variable d'état et $1_{\omega}$ la fonction caractéristique de $\omega$. L'opérateur de Kalman, $\mathcal{K}$ est défini dans $(3)$ et $\left(\mathcal{K}^{*}\right)$ désigne son adjoint dans $L^{2}(\Omega)^{n}$. Notre principal résultat est :

Théorème Le système (1) est contrôlable aux trajectoires en tout temps $T>0$ si et seulement si

$$
\operatorname{Ker}\left(\mathcal{K}^{*}\right)=\{0\} .
$$

La condition nécessaire se vérifie aisément. La démonstration de la partie suffisante se fait en deux étapes. Dans un premier temps, la définition de l'opérateur $\mathcal{K}$ permet de transformer le système adjoint de (1) en un système en cascade pour lequel on démontre une inégalité d'observabilité de type Carleman. Celle-ci est obtenue via une inégalité de Carleman pour des opérateurs paraboliques d'ordre $n$ et une inégalité d'observabilité pour des systèmes d'ordre 2. Dans une deuxième partie, on conclut en revenant au système initial grâce à (4).

Le contrôle aux trajectoires de tels systèmes par le bord est ouvert.

\section{Introduction}

Controllability of linear differential systems is well-known. In particular we have at our disposal the famous Kalman rank condition (see for example [8, Chapter 2, p. 35]), that is to say, if $A \in \mathcal{L}\left(\mathbb{R}^{n}\right)$, $B \in \mathcal{L}\left(\mathbb{R}^{m}, \mathbb{R}^{n}\right)$, then the system $Y^{\prime}=A Y+B u$ is controllable at time $T>0$ if and only if rank $[A \mid B]=$ rank $\left[B, A B, \ldots, A^{n-1} B\right]=n$. Our main goal is to extend this algebraic condition to partial differential systems. For $n, m \in \mathbb{N}^{*}$ and $T>0$, we consider the following $n \times n$ parabolic system

$$
\left\{\begin{array}{l}
\partial_{t} y=(M R+A) y+B v 1_{\omega} \text { in } \Omega_{T}=\Omega \times(0, T), \\
y=0 \text { on } \Sigma_{T}=\partial \Omega \times(0, T), \quad y(\cdot, 0)=y_{0}(\cdot) \text { in } \Omega,
\end{array}\right.
$$

where $\Omega \subset \mathbb{R}^{d}$ is a bounded open set with a $C^{2}$-boundary $\partial \Omega, \omega \subset \Omega$ is an open subset, $T>0$, $M=P D P^{-1}$, where $D=\operatorname{diag}\left(d_{i i}\right)_{n \times n} \in \mathcal{L}\left(\mathbb{R}^{n}\right), R$ is the second order elliptic self adjoint operator given by

$$
R=\Sigma_{i, j} \partial_{i} r_{i j}(x) \partial_{j}+c(x)
$$

with $r_{i j} \in C^{1}(\bar{\Omega}), c \in L^{\infty}(\Omega) . A=\left(a_{i j}\right)_{1<i, j \leq n} \in \mathcal{L}\left(\mathbb{R}^{n}\right), B \in \mathcal{L}\left(\mathbb{R}^{m}, \mathbb{R}^{n}\right), v \in L^{2}\left(\Omega_{T}\right)^{m}\left(\Omega_{T}=\right.$ $(0, T) \times \Omega), y_{0}=\left(y_{0, i}\right)_{1 \leq i \leq n} \in L^{2}(\Omega)^{n}$. In $(1), y=\left(y_{i}\right)_{1 \leq i \leq n}$ is the state variable while $1_{\omega}$ denotes the characteristic function of the open subset $\omega$. We assume that $d_{i i}>0, \forall i \in\{1, \ldots, n\}$. The exact controllability to zero reads as follows: For given $T>0$ and $y_{0} \in L^{2}(\Omega)^{n}$ can we find $v \in L^{2}\left(\Omega_{T}\right)^{m}$ such that the corresponding solution $y$ of $(1)$ satisfies $y(T)=0$ a.e. in $\Omega$ ? In the linear case, this property is equivalent to the controllability to trajectories. There are few results on null controllability of system (1) when $n>1$ and most of them are proved for $n=2$ : see, for instance, [11], [1], [2] and [5]. Also see [6] where the authors provide a null controllability result for a general cascade parabolic system of $n$ equations.

To be clear, we will present our result in the case where $M=D$ (i.e., $P \equiv I_{d}$ ) and $R=\Delta$. All the proofs can be adapted to the general case. Let us now introduce the Kalman operator. If we denote by 
$L:=D \Delta+A$ with $D(L)=D(\Delta)^{n}=\left(H^{2}(\Omega) \cap H_{0}^{1}(\Omega)\right)^{n}$, then the Kalman operator associated with $(L, B)$ is the operator matrix

$$
\left\{\begin{array}{l}
\mathcal{K}:=[L \mid B]=\left[L^{n-1} B, L^{n-2} B, \ldots, L B, B\right]: D(\mathcal{K}) \subset L^{2}(\Omega)^{n m} \rightarrow L^{2}(\Omega)^{n}, \text { with } \\
D(\mathcal{K}):=\left\{u \in L^{2}(\Omega)^{n m}: \mathcal{K} u \in L^{2}(\Omega)^{n}\right\}
\end{array}\right.
$$

Our main result is the following:

Theorem 1.1 System (1) is exactly controllable to trajectories at any time $T$ if and only if the Kalman operator $\mathcal{K}$ satisfies

$$
\operatorname{Ker}\left(\mathcal{K}^{*}\right)=\{0\} .
$$

Of course, this result is still true if $\Delta$ is replaced by $R$ (defined in (2)). If $\left(\lambda_{p}\right)_{p \in \mathbb{N}^{*}}$ is the sequence of eigenvalues of $-\Delta$ with Dirichlet boundary conditions, we consider the matrices $L_{p}=-\lambda_{p} D+A \in \mathcal{L}\left(\mathbb{R}^{n}\right)$ and $\mathcal{K}_{p}=\left[L_{p} \mid B\right]=\left[L_{p}^{n-1} B, \ldots, L_{p} B, B\right] \in \mathcal{L}\left(\mathbb{R}^{n m}, \mathbb{R}^{n}\right)$, for every $p \in \mathbb{N}^{*}$. It is not difficult to see that (4) is equivalent to

$$
\operatorname{rank} \mathcal{K}_{p}=n, \quad \forall p \in \mathbb{N}^{*} .
$$

Note that either (5) reduces to a finite number of conditions or it is never satisfied.

Remark 1 (i) The closest result of this kind we know is due to H. Leiva [9] which gives a necessary and sufficient condition for the approximate controllability of (1) in the case where $A \equiv 0, D$ is not diagonal and $B(x) \in \mathbb{R}^{n}(m \equiv 1)$.

(ii) A necessary and sufficient condition (if it exists) of controllability to trajectories in the case of controls acting on a subset of the boundary is still open for such systems.

(iii) To our knowledge, the null controllability problem of (1) when the operators are time dependent is still open. We will address in details this question in a forthcoming paper. On the other hand, the case in which $A, B$ depend on $x$ seems to be much more complicated. A necessary and sufficient condition for the controllability of (1) is open even if there are some partial results (see [11], [2], [1], [5], [6]). The methods used in this paper would hardly provide general results for non-autonomous systems since the proofs take advantage in a crucial way of the fact that the matrices considered are constant in time and space variables.

\section{Sketch of the proof of Theorem 1.1}

Controllability of System (1) is equivalent (see for instance $[10],[3], \ldots$ ) to the existence of a positive constant $C$ such that, for every $\varphi_{0} \in L^{2}(\Omega)^{n}$, the solution $\varphi \in C^{0}\left([0, T] ; L^{2}(\Omega)^{n}\right)$ of the following adjoint system

$$
\left\{\begin{array}{l}
-\partial_{t} \varphi=\left(D \Delta+A^{*}\right) \varphi \text { in } \Omega_{T} \\
\varphi=0 \text { on } \Sigma_{T}, \quad \varphi(\cdot, T)=\varphi_{0}(\cdot) \text { in } \Omega
\end{array}\right.
$$

(where we have used that $D^{*}=D$ ) satisfies the observability inequality (with the notation $\omega_{T}=\omega \times(0, T)$ )

$$
\|\varphi(\cdot, 0)\|_{L^{2}(\Omega)}^{2} \leq C \iint_{\omega_{T}}\left|B^{*} \varphi(x, t)\right|^{2} d x d t
$$

We will need, in an essential way, the following properties of the Kalman operator: 
Proposition 2.1 (i) If $u \in D\left(\Delta^{n-1}\right)^{n m}$ then $\mathcal{K} u \in L^{2}(\Omega)^{n}$ and $\|\mathcal{K} u\|_{L^{2}(\Omega)^{m}} \leq C\|u\|_{D\left(\Delta^{n-1}\right)^{n m}}$.

(ii) Assume $\operatorname{Ker}\left(\mathcal{K}^{*}\right)=\{0\}$. Let $r \in \mathbb{R}$. There exists $C>0$ such that for every $\varphi \in L^{2}(\Omega)^{n}$ satisfying $\mathcal{K}^{*} \varphi \in D\left(\Delta^{r}\right)^{n m}$, one has :

$$
\|\varphi\|_{D\left(\Delta^{r-\left(n^{2}-1\right) / 2}\right)^{n}}^{2} \leq C\left\|\Delta^{r} \mathcal{K}^{*} \varphi\right\|_{L^{2}(\Omega)^{n m}}^{2} .
$$

\subsection{Necessary condition}

Suppose that $\operatorname{Ker}\left(\mathcal{K}^{*}\right) \neq\{0\}$. It follows from (5) that there exists $p_{0} \in \mathbb{N}^{*}$ such that $0 \in \sigma\left(\mathcal{K}_{p_{0}}\right)$. Therefore, thanks to Kalman's rank condition applied to the pair $\left(-\lambda_{p_{0}} D+A, B\right)$, there exists a non zero solution $z_{p_{0}}(t) \in \mathbb{R}^{n}$ of the adjoint system associated with $\left(-\lambda_{p_{0}} D+A, B\right)$

$$
\left\{\begin{array}{l}
-z^{\prime}=\left(-\lambda_{p_{0}} D+A^{*}\right) z \text { in }(0, T) \\
z(0)=z_{0}^{p} \in \mathbb{R}^{n}=0
\end{array}\right.
$$

satisfying $B^{*} z_{p_{0}}(t)=0, \forall t \in[0, T]$. Then, letting $\varphi_{0}=z_{p_{0}}(T) \phi_{p_{0}}$, where $\phi_{p_{0}}$ is an eigenfunction associated with $\lambda_{p_{0}}$, it is easy to check that $\varphi(t, x)=z_{p_{0}}(t) \phi_{p_{0}}(x)$ is the solution of $(6)$. It is non zero and satisfies $B^{*} \varphi(t)=0, \forall t \in[0, T]$. Obviously this solution does not satisfy the observability inequality (7), and thus (1) is not null controllable.

\subsection{Sufficient condition}

2.2.1. A Carleman inequality for a parabolic equation of order $n$

Let us denote by $\mathcal{D}=\cap_{p \geq 0} D\left(\Delta^{p}\right)$. If we set $X=\mathcal{D}^{n}$ then, one has:

Proposition 2.2 If $\varphi=\left(\varphi_{1}, \cdots, \varphi_{n}\right)^{*}$ is the solution to (6) corresponding to $\varphi_{0} \in X$, then

$$
\operatorname{det}\left(I_{d} \partial_{t}+D \Delta+A^{*}\right) \varphi_{i}=0 \quad \text { in } \quad \Omega_{T}, \quad \forall i: 1 \leq i \leq n
$$

This proposition can be deduced as in the case of an ordinary differential system (see $[7, \S 6.4$, p. 144]).

In order to state our second result, let us introduce some additional notations. If $s, \tau \in \mathbb{R}$, we define the following functionals

$$
\left\{\begin{array}{l}
I(s, \varphi)=\tau^{s-1} \iint_{\Omega_{T}} \rho^{s-1} e^{-2 \tau \alpha}\left(\left|\varphi_{t}\right|^{2}+|\Delta \varphi|^{2}+\tau^{2} \rho^{2}|\nabla \varphi|^{2}+\tau^{4} \rho^{4}|\varphi|^{2}\right) d x d t, \\
\mathcal{I}(s, \phi)=I(s+3(n-1), \phi)+\sum_{p=1}^{n-1} \sum_{1 \leq i_{1}<\ldots<i_{p} \leq n} I\left(s+3(n-p-1), P_{i_{p}} \ldots P_{i_{1}} \phi\right),
\end{array}\right.
$$

where $\rho=\rho(x, t)$ and $\alpha=\alpha(x, t)$ are suitable weight functions defined in $\Omega_{T}$ and where the operator $P_{i}$ is given by $P_{i} \equiv \partial_{t}+d_{i} \Delta(1 \leq i \leq n)$. We will obtain the proof of the observability inequality $(7)$ as a consequence of the following result:

Theorem 2.1 Let us assume that $n, k_{1}, k_{2} \in \mathbb{N}$ and $s_{0} \in \mathbb{R}$ are given. Then, there exist two positive functions $\alpha_{0}, \alpha_{1} \in C^{2}(\bar{\Omega})$ (only depending on $\Omega$ and $\omega$ ), two positive constants $C$ and $\sigma$ (only depending on $\Omega, \omega, n, s_{0}, k_{1}$ and $\left.k_{2}\right)$ and $r_{0}=r_{0}(n) \in \mathbb{N}$ such that

$$
\sum_{i=0}^{k_{1}} \sum_{j=0}^{k_{2}} \mathcal{I}\left(s_{0}-4(i+j), \Delta^{i} \partial_{t}^{j} \phi\right) \leq C \tau^{s_{0}+r_{0}} \iint_{\omega_{T}} \rho^{s_{0}+r_{0}} e^{-2 \tau \alpha}|\phi|^{2}, \quad \forall \tau \geq \sigma\left(T+T^{2}\right),
$$


for every $\phi \in C^{\infty}((0, T) ; \mathcal{D})$ solution to (8). In (9), the functions $\alpha$ and $\rho$ are given by $\alpha(x, t)=$ $\alpha_{0}(x) / t(T-t)$ and $\rho(x, t)=\alpha_{1}(x) / t(T-t)$.

Sketch of the proof: We will present a sketch of the proof in the particular case in which $k_{1} \equiv k_{2} \equiv 0$. Let us fix $s_{0} \in \mathbb{R}$ and $\phi \in C^{\infty}((0, T) ; \mathcal{D})$ a solution to (8). If we develop the determinant in (8) we deduce that $\phi$ also satisfies

$$
\left(P_{n} \cdots P_{1}+\sum_{p=2}^{n-1} \sum_{1 \leq i_{1}<\ldots<i_{p} \leq n} \alpha_{i_{1}, \ldots, i_{p}} P_{i_{1}} \ldots P_{i_{p}}+\sum_{i=1}^{n} \alpha_{i} P_{i}+\alpha\right) \phi=0 \text { in } \Omega_{T}
$$

where the coefficients $\alpha_{i_{1}, \ldots, i_{p}}, \alpha_{i}$ and $\alpha$ are real numbers that only depend on the coefficients of $D$ and $A$. If we perform the following change of variables $\psi_{1}=\phi, \psi_{i}=P_{i-1} \psi_{i-1}=\left(\partial_{t}+d_{i-1} \Delta\right) \psi_{i-1}$, for $2 \leq i \leq n$, it follows from $(10)$ that $\left(\psi_{1}, \ldots, \psi_{n}\right)^{*}$ solves the cascade parabolic system

$$
\left\{\begin{array}{l}
\left(\partial_{t}+d_{1} \Delta\right) \psi_{1}=\psi_{2} \text { in } \Omega_{T} \\
\left(\partial_{t}+d_{2} \Delta\right) \psi_{2}=\psi_{3} \text { in } \Omega_{T} \\
\cdots \\
\left(\partial_{t}+d_{n} \Delta\right) \psi_{n}=f \text { in } \Omega_{T} \\
\psi_{i}=0, \text { on } \Sigma_{T}
\end{array}\right.
$$

with

$$
f=f(\phi)=-\sum_{p=2}^{n-1} \sum_{1 \leq i_{1}<\ldots<i_{p} \leq n} \alpha_{i_{1}, \ldots, i_{p}} P_{i_{1}} \ldots P_{i_{p}} \phi+\sum_{i=1}^{n} \alpha_{i} P_{i} \phi+\alpha \phi \in L^{2}\left(\Omega_{T}\right) .
$$

Thanks to the cascade structure of system (11) and following the ideas in [6] (also see [5]), we can prove that there exist $r_{0}=r_{0}(n) \in \mathbb{N}$ and positive constants $\sigma=\sigma\left(\Omega, \omega, s_{0}, n\right)$ and $C=C\left(\Omega, \omega, s_{0}, n\right)$ such that

$$
\sum_{i=1}^{n} I\left(s_{0}+3(n-i), \psi_{i}\right) \leq C\left(\tau^{s_{0}+r_{0}} \iint_{\omega_{T}} \rho^{s_{0}+r_{0}} e^{-2 \tau \alpha}\left|\psi_{1}\right|^{2} d x d t+\tau^{s_{0}} \iint_{\Omega_{T}} \rho^{s_{0}} e^{-2 \tau \alpha}|f|^{2} d x d t\right),
$$

for every $\tau \geq \sigma\left(T+T^{2}\right)$. The key point in the proof of this last inequality is the following global Carleman estimate for the heat equation

$$
I\left(s_{0}, \varphi\right) \leq C\left(\tau^{s_{0}} \iint_{\Omega_{T}} \rho^{s_{0}} e^{-2 \tau \alpha}\left|\varphi_{t} \pm c \Delta \varphi\right|^{2} d x d t+\tau^{s_{0}+3} \iint_{\omega_{T}} \rho^{s_{0}+3} e^{-2 \tau \alpha}|\varphi|^{2} d x d t\right),
$$

valid for any $\tau \geq \sigma\left(T+T^{2}\right)$ and any function $\varphi \in L^{2}\left(0, T ; H_{0}^{1}(\Omega)\right)$ such that $\varphi_{t} \pm c \Delta \varphi \in L^{2}\left(\Omega_{T}\right)($ see $[4])$.

Now, if $\Pi$ denotes any permutation of $\{1,2, \ldots, n\}$, one can consider the new functions $\widetilde{\psi}_{1}=\phi, \widetilde{\psi}_{i}=$ $P_{\Pi(i-1)} \widetilde{\psi}_{i-1}$, deducing that $\widetilde{\psi}=\left(\widetilde{\psi}_{1}, \ldots, \widetilde{\psi}_{n}\right)^{*}$ is a solution to system $(11)$ with $d_{\Pi(i)}$ instead of $d_{i}$. We can apply the previous argument, deducing that (13) holds for $\widetilde{\psi}$. Finally, using the definition of $f$ (see (12)), we infer (9) in the particular case $k_{1}=k_{2}=0$. This ends the proof. 


\subsubsection{Use of the Kalman condition}

From Proposition 2.1 we deduce that $X \subset D\left(\mathcal{K}^{*}\right)$. We also have that $X$ is dense in $L^{2}(\Omega)^{n}$ and, since the constant $C$ in (7) is independent of $\varphi_{0}$, it is sufficient to prove inequality (7) for $\varphi_{0} \in X$. Under this assumption, the corresponding solution to $(6)$ satisfies $\varphi \in C^{\infty}((-\infty, T) ; X)$ and

$$
\mathcal{K}^{*} \varphi(\cdot, t) \equiv\left((-1)^{n-1} \partial_{t}^{n-1} w,(-1)^{n-2} \partial_{t}^{n-2} w, \cdots,-\partial_{t} w, w\right)^{*}(\cdot, t), \quad \forall t \in[0, T),
$$

where $w=B^{*} \varphi$. From Proposition 2.1 and using inequality (8) for $w=B^{*} \varphi$, if $\operatorname{Ker}\left(\mathcal{K}^{*}\right)=\{0\}$, the following inequality holds:

$$
\begin{aligned}
\|\varphi(\cdot, 0)\|_{L^{2}(\Omega)^{n}}^{2} & \leq C \int_{T / 4}^{3 T / 4}\|\varphi(\cdot, t)\|_{L^{2}(\Omega)^{n}}^{2} d t \leq C \int_{T / 4}^{3 T / 4}\left\|\Delta^{(n-1)(2 n-1)} \mathcal{K}^{*} \varphi(\cdot, t)\right\|_{L^{2}(\Omega)^{n m}}^{2} d t \\
& \leq C \sum_{j=0}^{n-1} \iint_{\Omega_{T}} e^{-2 \tau \alpha}\left|\Delta^{(n-1)(2 n-1)} \partial_{t}^{j} w_{\ell}\right|^{2} d x d t .
\end{aligned}
$$

On the other hand, thanks to Proposition 2.2, we can apply Theorem 2.1 with $k_{1}=(n-1)(2 n-1)$, $k_{2}=n-1$ and $s_{0}=8 n(n-1)$ to each component $w_{\ell}=\left(B^{*} \varphi\right)_{\ell}, 1 \leq \ell \leq m$, obtaining

$$
\sum_{j=0}^{n-1} \iint_{\Omega_{T}} e^{-2 \tau \alpha}\left|\Delta^{(n-1)(2 n-1)} \partial_{t}^{j} w_{\ell}\right|^{2} d x d t \leq C \tau^{r_{0}+8 n(n-1)} \iint_{\omega_{T}} e^{-2 \tau \alpha} \rho^{r_{0}+8 n(n-1)}\left|B^{*} \varphi\right|^{2} d x d t,
$$

which is valid for $\tau \geq \sigma\left(T+T^{2}\right)$. Choosing $\tau=\sigma\left(T+T^{2}\right)$ and combining these two last inequalities we can infer that inequality (7) is satisfied. Therefore, system (1) is null controllable at time $T$. This ends the proof of Theorem 1.1.

\section{References}

[1] F. Ammar Khodja, A. Benabdallah, C. Dupaix, Null-controllability of some reaction-diffusion systems with one control force, J. Math. Anal. Appl., 320 (2006), no. 2, 928-943.

[2] F. Ammar-Khodja, A. Benabdallah, C. Dupaix, I. Kostine, Null controllability of some systems of parabolic type by one control force, ESAIM Control Optim. Calc. Var., vol. 11 (2005), no. 3, 426-448.

[3] E. Fernández-Cara, E. Zuazua, The cost of approximate controllability for heat equations: The linear case, Advances in Differential Equations 5 (2000), 4-6, 465-514.

[4] A. Fursikov and O. Yu. Imanuvilov, Controllability of Evolution Equations, Lecture Notes Ser. 34, Seoul National University, Korea, 1996.

[5] M. González-Burgos, R. Pérez-García, Controllability results for some nonlinear coupled parabolic systems by one control force, Asymptotic Analysis 46 (2006) 123-162.

[6] M. González-Burgos, L. de Teresa, Controllability results for cascade systems of $m$ coupled parabolic PDEs by one control force, submited to Systems Control Lett.

[7] E. L. Ince, Ordinary Differential Equations, Dover Publications, New York, 1944.

[8] R. E. Kalman, P. L. Falb \& M. A. Arbib, Topics in mathematical control theory, McGraw-Hill Book Co., New YorkToronto, Ont.-London 1969.

[9] H. Leiva, Controllability of a system of parabolic equations with non-diagonal diffusion matrix. IMA J. Math. Control Inform. 22 (2005), no. 2, 187-199.

[10] D. L. Russell Controllability and stabilizability theory for linear partial differential equations: Recent progress and open questions SIAM Review, Vol. 20 (1978), no. 4., 639-739.

[11] L. de Teresa, Insensitizing controls for a semilinear heat equation, Comm. PDE, 25 (2000), no. 1-2, 39-72. 